\title{
Discovery of fructans in Archaea
}

Onur Kırtel ${ }^{1}$, Eveline Lescrinier², Wim Van den Ende3, and Ebru Toksoy Öner ${ }^{1, *}$

${ }^{1}$ IBSB-Industrial Biotechnology and Systems Biology Research Group, Department of Bioengineering,

Marmara University, Göztepe Campus, Istanbul, Turkey

2Medicinal Chemistry, Rega Institute for Medical Research, KU Leuven, Leuven, Belgium.

3Laboratory of Molecular Plant Biology, KU Leuven, Leuven, Belgium

Corresponding author at: IBSB-Industrial Biotechnology and Systems Biology Research Group, Department of Bioengineering, Marmara University, Göztepe Campus, Istanbul, Turkey

E-mail address: ebru.toksoy@marmara.edu.tr

\begin{abstract}
Fructans are fructose-based oligo- and polysaccharides derived from sucrose that occur in a plethora of Eubacteria and plants. While fructan-producing (fructanogenic) Eubacteria are abundant in hypersaline environments, fructan production by Archaea has never been reported before. Exopolysaccharides accumulated by various Archaea from the Halobacteria class (belonging to the genera of Halomicrobium, Haloferax and Natronococcus) originating from dif-

Abbreviations: EPSs, extracellular polysaccharides; FTs, fructosyltransferases; GH32, glycoside hydrolase 32 family; GH68, glycoside hydrolase 68 family, GH-J, glycoside hydrolase J clan; gHSQC, gradient Heteronuclear Single Quantum Coherence; HMBC, Heteronuclear Multiple Bond Correlation; HSQC, Heteronuclear Single Quantum Coherence; HPAEC-IPAD, High Performance Anion Exchange Chromatography with Integrated Pulsed Amperometric Detector; NOESY, Nuclear Overhauser Spectroscopy; Suc, sucrose; TOCSY, Total Correlated Spectroscopy.
\end{abstract}


ferent locations on Earth were structurally characterized as either levans or inulins with varying branching degrees (10\% - 16\%). Thus, we show for the first time in the literature that fructans are produced in all three domains of life, including Archaea. This proof of concept will not only provide insight into Archaeal glycans and evolution, but it may also open new frontiers for innovative strategies to overcome the ever-increasing threat of excessive salinization.

Keywords: Fructan; Archaea; halophiles; salinity; inulin; levan

\section{Introduction}

Fructans are a diverse class of sucrose (Suc)-derived oligo- and polyfructosyl saccharides that naturally occur in a wide variety of Eubacteria and plants, hereafter referred to as fructanogenic Eubacteria and plants, and they are believed to act as multifunctional components due to their key roles in biofilms (Koczan, McGrath, Zhao, \& Sundin, 2009; Dogsa, Brloznik, Stopar, \& Mandic-Mulec, 2013), prebiotic (Adamberg et al., 2015; Lei et al., 2017) and immunomodulatory effects (Peshev \& Van den Ende, 2014; Xu et al., 2016) and roles in plant stress responses (Tarkowski, Van de Poel, Höfte, \& Van den Ende, 2019; Wei et al., 2017). There are mainly two structural forms of fructans in nature: levans ( $\beta-2,6$ linkages between fructofuranosyl units on the polymer backbone with occasional branching at $\beta-2,1$ positions) and inulins $(\beta-2,1$ linked fructofuranosyl units on the backbone with possible branching points on $\beta-2,6$ positions). In Eubacteria, fructans are synthesized as extracellular oligo- and polysaccharides (EPSs) of levan and inulin types by the catalytic action of fructosyltransferase (FT) enzymes levansucrases (EC 2.4.1.10) and inulosucrases (EC 2.4.1.9), respectively. While levans are synthesized by a plethora of both Gram-negative and Gram-positive Eubacteria, so far microbial inulin synthesis has only been observed in some Gram-positive species (mostly lactic acid bacteria; Zannini, Waters, Coffey, \& Arendt, 2016; Kralj et al., 2018). Contrary to Eubacteria, in plants, fructans are accumulated intracellularly. In addition to levan- and inulin-type fructans, highly branched neo-fructans (e.g. agavins in Agave species) and graminans (e.g. in cereals) occur in plants, which are produced by at least three different 
types of plant FTs. (Avila-de Dios, Gomez-Vargas, Damian-Santos, \& Simpson, 2015; Verspreet et al., 2015). It should be noted that bacterial and plant fructan types greatly differ in terms of their degree of polymerization (DP), such that on average, bacterial fructan DPs are several orders higher as compared to plants (Toksoy Öner, Hernández, \& Combie, 2016). Some fungi can accumulate fructans as well (Choukade \& Kango, 2019; Zhang et al., 2017), but fructans are completely absent in animals.

Hypersaline environments are habitats with salinity values greater than that of oceans and seas, and are predominantly inhabited by halophilic Archaea, followed by Eubacteria, viruses and some Eukarya (Oren, 2008; Loukas, Kappas, \& Abatzopoulos, 2018). Our recent findings revealed that hypersaline environments are rich in a wide variety of fructanogenic Eubacteria (Kırtel, Versluys, Van den Ende, \& Toksoy Öner, 2018). However, the presence of fructans has never been reported in Archaea so far. Although both the CAZy database (http://www.cazy.org) and preliminary bioinformatic studies carried out in our previous study (Kırtel, Versluys, Van den Ende, \& Toksoy Öner, 2018) suggested the presence of putative Archaeal fructan biosynthetic genes, the production of fructans by Archaea has never been experimentally demonstrated.

Bacterial FTs belong to the GH68 family enzymes. These enzymes use Suc as their preferential fructosyl donor; hydrolyzing it and transferring the resulting fructosyl moiety to another Suc, with successive transfers resulting in an elongated chain of fructan while releasing glucose. In plants, however, synthesis of fructans is accomplished by a different set of FTs belonging to GH32 family, which together with the GH68 family form the GH-J clan of enzymes. GH32 family enzymes are also present in many Eubacteria and fungi, with the bestknown ones being invertases/ $\beta$-fructofuranosidases (hydrolyzing Suc, but also showing some FT activity at elevated Suc concentrations), levanases (hydrolyze levans) and inulinases (hydrolyze inulins). All GH-J clan enzymes carry a 5 -bladed $\beta$ propeller domain with a deep central cavity where substrate is bound. In addition to the 5-bladed $\beta$ propeller domain, GH32 family enzymes also carry a $\beta$-sandwich domain composed of six $\beta$-strands (Lammens et al., 2009). 
Archaea form one of the three domains of life on Earth along with Eubacteria and Eukarya (Woese, Kandler, \& Wheelis, 1990), and they were originally associated exclusively with extremely saline or hot environments. However, recent studies suggest that they are much more prevalent than previously thought, with novel Archaeal species being constantly discovered in human body (Gaci, Borrel, Tottey, O’Toole, \& Brugère, 2014; Borrel et al., 2017), soil (Orellana, Chee-Sanford, Sanford, Löffler, \& Konstantinidis, 2018) , the plant apoplast (Hardoim et al., 2015) and many other mesophilic environments. Their functions in those habitats are still largely undiscovered and present an exciting research area. Moreover, not long ago it was suggested in a comprehensive study that Archaea actually share a more recent last common ancestor with Eukarya than Eubacteria (Zaremba-Niedzwiedzka et al. 2017), making them even more intriguing organisms to study to better understand the evolution of life on Earth as a whole. A recent work revealed that the genetic structures of Halobacteria (also known as Haloarchaea) have been under significant influence of Eubacteria via gene transfers throughout their evolution, enhancing their carbohydrate metabolism drastically by creating chimeric protein families which also include GH32-like genes (Méheust et al., 2018). Those adaptations may have helped Halobacteria to adopt an aerobic lifestyle by allowing them to utilize a wide range of carbohydrates via aerobic respiration. Also, it is possible that other aerobiosis-related genes and GH-J clan genes were acquired from Eubacteria at the same time, which may have rendered Halobacteria more successful in the environment. What's intriguing is that the putative GH-J clan genes appear only in the halophilic class of Halobacteria, and not in any other class of Archaea. Preservation of GH-J-like genes only in the Halobacteria class of all Archaeal lineages raises provoking questions: are those pseudogenes that have no effect on the phenotype, or are they translated and expressed as active enzymes? If so, what are the functions of fructans in hypersaline environments? Are they simply parts of the biofilms produced by these microorganisms? Considering their excellent water absorption capabilities (Kang et al., 2009), do fructans help those microorganisms survive extreme desiccation periods typically seen in hypersaline environments? To date, these questions have never been investigated, and they present a completely novel aspect regarding life under extreme salinity as a whole. 
Here, we sought for evidence that fructans occur in Halobacterial species. Three type strains harboring putative GH-J clan genes from three different continents (Halomicrobium mukohataei (Hmc. mukohataei) DSM 12286 from Argentina, Haloferax prahovense (Hfx. prahovense) DSM 18310 from Romania, and Natronococcus jeotgali (Ncc. jeotgali) DSM 18795 from Korea) together with four Halomicrobium strains isolated from crude salt samples of a Turkish saltern (Tuz Lake) were investigated for their fructanogenic traits, and they were all shown to be able to synthesize different types of fructans. This is the first report in the literature that demonstrates the presence of fructans in Archaea.

\section{Materials and Methods}

\subsection{Origin of strains}

Several Archaeal colonies were isolated from crude salt samples (Estuz Ltd., Izmir, Turkey) originating from Tuz Lake, Turkey $\left(38^{\circ} 50^{\prime} \mathrm{N} 35^{\circ} 20^{\prime} \mathrm{E}\right)$. The other strains were purchased from DSMZ GmbH (Germany), namely Halomicrobium mukohataei DSM 12286 isolated from Salinas Grandes, Argentina (Ihara, Watanabe, \& Tamura, 1997; Oren, Elevi, Watanabe, Ihara, \& Corcelli, 2002), Haloferax prahovense DSM 18310 isolated from Telega Lake, Romania (Enache, Itoh, Kamekura, Teodosiu, \& Dumitru, 2007), and Natronococcus jeotgali DSM 18795 isolated from fermented shrimp in Korea (Roh et al., 2007). These particular strains were selected because of the presence of putative GH68 family genes in their genomes (Kirtel et al., 2018a).

\subsection{Taxonomic classification of Tuz Lake isolates}

For their genetic identification, Tuz Lake strains were grown in DSMZ Medium 372 under conditions mentioned above. After a week of incubation, genomic DNA of the isolates were extracted using DNeasy® UltraClean $\AA$ Microbial Kit from QIAGEN, following manufacturer's instructions. Extracted genomic DNA samples were sent to Macrogen Ltd. (Amsterdam, The Netherlands) for $16 \mathrm{~S}$ ribosomal RNA gene sequencing. The universal-archaeal primer 21F (5' TCCGGTTGATCCYGCCGG 3') and the reverse primer 1492R (5' TACGGY- 
TACCTTGTTACGACTT 3') were used. Sequence chromatograms were refined with Chromas (v2.6.5) software.

\subsection{Isolation, media and culture conditions}

For the isolation of Halobacterial strains from Tuz Lake, crude salt samples were dissolved in a sterile medium at a final concentration of $250 \mathrm{~g} / \mathrm{L}$, with the following final composition (g/L): $\mathrm{MgSO}_{4} \cdot 7 \mathrm{H}_{2} \mathrm{O}, 20 ; \mathrm{KCl}, 2$; trisodium citrate, 3 ; casamino acids, 7.5; yeast extract, 1.0; and $\mathrm{FeSO}_{4} .7 \mathrm{H}_{2} \mathrm{O}, 0.0023 . \mathrm{pH}$ was adjusted to 7.6 under sterile conditions (Sehgal \& Gibbons, 1960). $50 \mathrm{~mL}$ of media were incubated in $250 \mathrm{~mL}$ Erlenmeyer flasks at $37^{\circ} \mathrm{C}$ and $180 \mathrm{rpm}$ for 10 days. At the end of this incubation period, $100 \mu \mathrm{L}$ of cell suspensions from the media were spread onto agar ( $15 \mathrm{~g} / \mathrm{L})$ plates with the same composition, supplied with $50 \mathrm{~g} / \mathrm{L}$ Suc for the selection of fructanogenic strains. Plates were incubated at $37^{\circ} \mathrm{C}$ for 2 weeks. Colonies exhibiting mucoid, convex, shiny phenotype were streaked onto fresh agar plates several times to obtain single colonies. Since no putative genes that take part in $\alpha$-glucan synthesis (GH70 family enzymes) could be found in any Halobacteria according to NCBI database, this morphology was considered as a strong indicator of fructan accumulation in sucrose-based media. Four single colonies were chosen randomly and used in the following experiments.

Type strains Hmc. mukohataei and $H f x$. prahovense were grown in DSMZ Medium 372 with the following composition (g/L): $\mathrm{NaCl}, 200 ; \mathrm{MgSO}_{4} \cdot 7 \mathrm{H}_{2} \mathrm{O}, 2 \mathrm{O} ; \mathrm{KCl}$, 2; trisodium citrate, 3; casamino acids, 5; yeast extract, 5; glutamic acid, $1 ; \mathrm{FeCl}_{2.4} \mathrm{H}_{2} \mathrm{O}, 0.036 ; \mathrm{MnCl}_{2.4} \mathrm{H}_{2} \mathrm{O}$, 0.00036; agar, for solid media 20; and Suc, for fructan production, 50. $\mathrm{pH}$ was adjusted to 7.0 under aseptic conditions after sterilization. For Ncc. jeotgali, DSMZ Medium 954 with the following composition was used (g/L): $\mathrm{NaCl}, 200 ; \mathrm{MgCl}_{2} .6 \mathrm{H}_{2} \mathrm{O}, 20$; TRIS, 12; $\mathrm{KCl}, 2$; $\mathrm{CaCl}_{2.2} \mathrm{H}_{2} \mathrm{O}, 0.2$; casamino acids, 5 ; yeast extract, 5; agar, for solid media, 20; Suc, for fructan production, 50. $\mathrm{pH}$ was adjusted to 7.4 under aseptic conditions after sterilization. In all cases, the media were sterilized at $121{ }^{\circ} \mathrm{C}$ for 15 minutes except for Suc, which was filter-steri- 
lized through $0.2 \mu \mathrm{m}$ syringe filters and added to media afterwards. All cultures were incubated at $37^{\circ} \mathrm{C}$ and $180 \mathrm{rpm}$ agitation rate throughout the experiments in this study. All strains were stored at $-80{ }^{\circ} \mathrm{C}$ in a $20 \%(\mathrm{w} / \mathrm{v}) \mathrm{NaCl}$ solution in $25 \%(\mathrm{v} / \mathrm{v})$ glycerol.

\subsection{Production and purification of fructans}

For fructan production, all strains were grown in their respective media supplemented with $50 \mathrm{~g} / \mathrm{L}$ Suc. Suc consumption throughout the incubation was recorded. Briefly, aliquots of $100 \mu \mathrm{l}$ from each culture were taken at regular intervals and centrifuged at 10,000 x $\mathrm{g}$ for 5 minutes. Supernatants were analyzed for their Suc concentrations via HPAEC-IPAD as described in section 2.5. When Suc depletion rate was no longer significant, cells were separated via centrifugation at 8,000 x $g$ for 20 minutes, and supernatants were dialyzed against several runs of distilled water at $4{ }^{\circ} \mathrm{C}$ through dialysis tubing with $13 \mathrm{kDa}$ cut-off. After dialysis, fructan polymers were precipitated with 1:1 (v:v) ethanol and left at $-20{ }^{\circ} \mathrm{C}$ overnight. Ethanol was removed via centrifugation at 8,000 x $\mathrm{g}$ for 10 minutes, and polysaccharide pellets were redissolved in ultrapure water. To pellet proteins, samples were boiled for 30 minutes and centrifuged at 8,000 $\mathrm{x} g$ for 20 minutes. Supernatants were passed through Dowex ${ }^{\mathrm{TM}}$ ion-exchange column (in Ac- form) to remove residual proteins, followed by chloroform extraction for fractions with relatively higher total protein content. For chloroform extraction of proteins, samples were mixed with 1:1 (v:v) chloroform and vortexed vigorously, and then centrifuged at 10,000 $\mathrm{x} g$ for 5 minutes for phase separation. The upper phase, or the flowthrough from Dowex ${ }^{\mathrm{TM}}$ column, was dried overnight at $30{ }^{\circ} \mathrm{C}$ in a Hetovac VR-I vacuum drier to obtain pure fructans.

\subsection{Chemical characterization of fructans}

Carbohydrate analyses of culture supernatants and purified EPSs were carried out via a Dionex ICS 5000 + HPAEC-IPAD (Thermo Scientific) system. Samples were diluted 100x in $20 \mu \mathrm{M}$ rhamnose, which was used as the internal standard, then were run for 45 minutes. 
The flow rate was $0.25 \mathrm{ml} / \mathrm{min}$, and the column used was Dionex CarboPac PA10o (2x50 $\mathrm{mm})$. Starting with $100 \%$ mobile phase A (90 $\mathrm{mM} \mathrm{NaOH})$, a gradient was used for mobile phase $\mathrm{B}$ ( $90 \mathrm{mM} \mathrm{NaOH}+0.5 \mathrm{M} \mathrm{NaOAc}$ ), increasing from o to $35 \%$ in 26 minutes, followed by a cleaning step with $100 \%$ mobile phase $\mathrm{B}$. $\mathrm{Ag} / \mathrm{AgCl}$ (reference) and $\mathrm{Au}$ electrodes were utilized for amperometric detection, using a carbohydrate quadruple waveform. The reference solution used for the identification and quantification of the samples was a standard with $10 \mu \mathrm{M}$ rhamnose (internal standard), D-glucose, D-fructose and Suc.

After their purification, EPSs obtained from Halobacterial cultures grown on Suc were analyzed for their monomeric sugar composition via acid hydrolysis in $60 \mathrm{mM} \mathrm{HCl}$ at $70{ }^{\circ} \mathrm{C}$ for 90 minutes, followed by carbohydrate analysis with HPAEC-IPAD. Inulin (Orafti ${ }^{\circledR} \mathrm{HP}$, plant origin, low DP) and Halomonas levan (levan produced via the recombinant levansucrase enzyme of Halomonas smyrnensis AAD6, high DP, Kirtel et al., 2018b) were used as control samples.

\subsection{NMR analysis}

To determine the linkage types of the purified EPSs, vacuum-dried pure fructan samples were dissolved in $0.5 \mathrm{ml} \mathrm{D}_{2} \mathrm{O}$ (99.8\%) prior to the measurement of Nuclear Magnetic Resonance (NMR) spectra that were recorded at Bruker Avance II $600 \mathrm{MHz}$ at $293 \mathrm{~K}$ equipped with $5 \mathrm{~mm}$ TCI HCN Z gradient cryoprobe. In one-dimensional proton spectra HOD signal was suppressed by applying presaturation during relaxation delay for $1 \mathrm{~s}$. Natural abundance gradient enhanced heteronuclear shift-correlated two-dimensional (2D) experiments (gHSQC) (Schleucher et al., 1994) were carried out using an F2 spectral width of $6000 \mathrm{~Hz}(1 \mathrm{H})$ and an $\mathrm{F} 1$ width of $5130 \mathrm{~Hz}\left({ }^{13 \mathrm{C})}\right.$; depending on concentration 4 to 220 time scans were accumulated with $4 \mathrm{~K}$ data points in $\mathrm{F} 2$ and 512 data points in F1 (256 in IBSBc sample due to low concentration), respectively. The structures of inulin (Orafti ${ }^{\circledR} \mathrm{HP}$ ) and Halomonas levan reference samples were confirmed by full assignment of ${ }^{1} \mathrm{H}$ and ${ }^{13} \mathrm{C}$ signals according to stan- 
dard methods relying on TOCSY, NOESY, HSQC and HMBC spectra (Vanhaecke, Van den Ende, Van Laere, Herdewijn, \& Lescrinier, 2006) .

\section{Results and Discussion}

\subsection{Chemical characterization of Archaeal EPSs}

Monomeric sugar compositions of purified EPSs from Archaea were determined with HPAEC-IPAD after acid hydrolysis. The results show that all seven EPS samples from different Archaeal strains were majorly composed of fructose with only trace amounts of glucose (Fig. 1), a typical profile for fructans. This was also verified with almost identical HPAECIPAD profiles obtained from acid-hydrolyzed inulin and levan control samples (not shown). Minor glucose signals in HPAEC-IPAD profiles indicate either the single terminal glucose residue originating from the initial Suc molecule (fructosyl acceptor) on which the polymerization process begins, or the internal glucose residue found in neo-fructan structures.

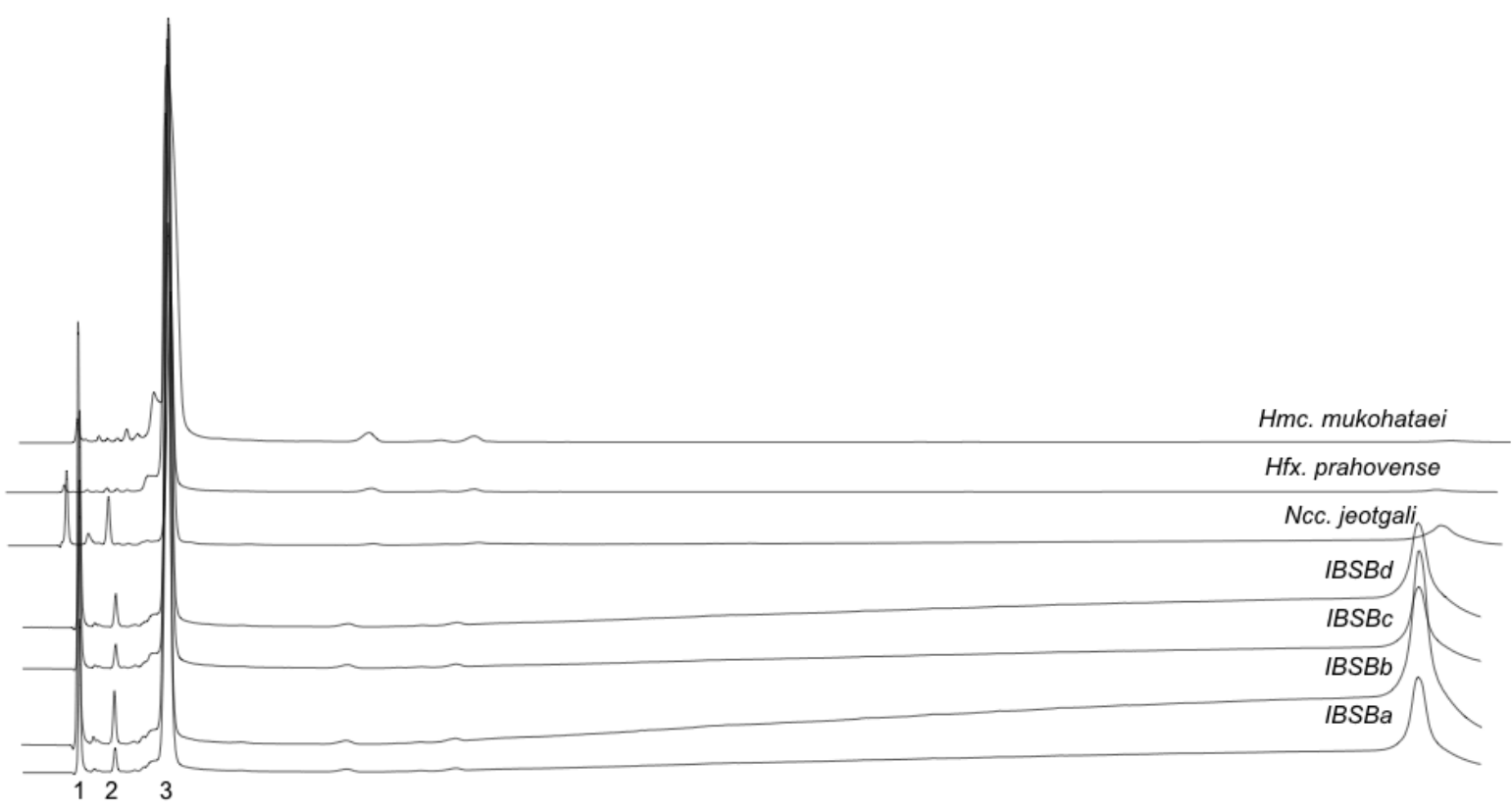


Fig. 1. Monomeric sugar compositions of Archaeal EPSs. HPAEC-IPAD chromatograms of acid-hydrolyzed Archaeal EPSs, showing their monomeric sugar compositions. Numbered peaks are: 1. rhamnose (internal standard), 2. glucose, 3. fructose. The peaks at the end of some chromatograms represent material retained on the column only after switching to $0.5 \mathrm{M} \mathrm{Na}$-acetate as mobile phase.

\subsection{NMR analysis}

Due to stereoelectronic effects of the different glycosidic bonds that interconnect fructoses in levan and inulin, the chemical shifts of their ${ }^{1} \mathrm{H}$ and ${ }^{13} \mathrm{C}$ signals each have a characteristic distribution. Despite signal overlap, one-dimensional ${ }^{1} \mathrm{H}$ spectra of both fructans are significantly different (Fig. 2, left). To resolve ${ }^{1} \mathrm{H}$ NMR signals and obtain ${ }^{13} \mathrm{C}$ chemical shifts on natural abundance samples at low concentration, 2D gHSQC spectra were recorded to obtain crosspeaks between hydrogens and the carbon to which they are directly bound. The characteristic downfield shift for carbons at linked positions to higher ppm is clearly visible in the overlayed gHSQC spectra of inulin and levan: in $\beta-2,1$ linked inulin C1 signals are downfield shifted compared with $\mathrm{C} 1$ in levan (+4.3 ppm) while in $\beta-2,6$ linked levan strong C6 signals in the main chain are downfield shifted compared with C6 in inulin $(+3.6 \mathrm{ppm})$. Due to their different glycosidic bonds, also chemical shifts of other carbons and protons in the fructofuranosyl ring slightly differ in inulin versus levan, though this effect is less pronounced. The C2 signal is not observable in a gHSQC since it is not directly bound to a hydrogen. A small degree of branching was determined in the reference sample of levan, allowing to determine chemical shift signatures for terminal fructoses that are involved in a single glycosidic bond and fructoses at branch points with both $\beta-2,1$ and $\beta-2,6$ linkages.

The $1 \mathrm{D}$ proton spectra of fructans isolated from Archaea resemble results on inulin in samples from Hmc. mukohataei DSM 12286, Hfx. prahovense DSM 18310, Ncc. jeotgali DSM 18795 and strain IBSBa, while strain IBSBb matches the spectrum of levan. Fructans from strain IBSBc and strain IBSBd have a $1 \mathrm{D}{ }^{1} \mathrm{H}$ spectrum with characteristics that could be 
attributed to levan as well as inulin, indicating the presence of both. gHSQC spectra of all Archaeal fructans can be found in Supplementary Materials 1-7.
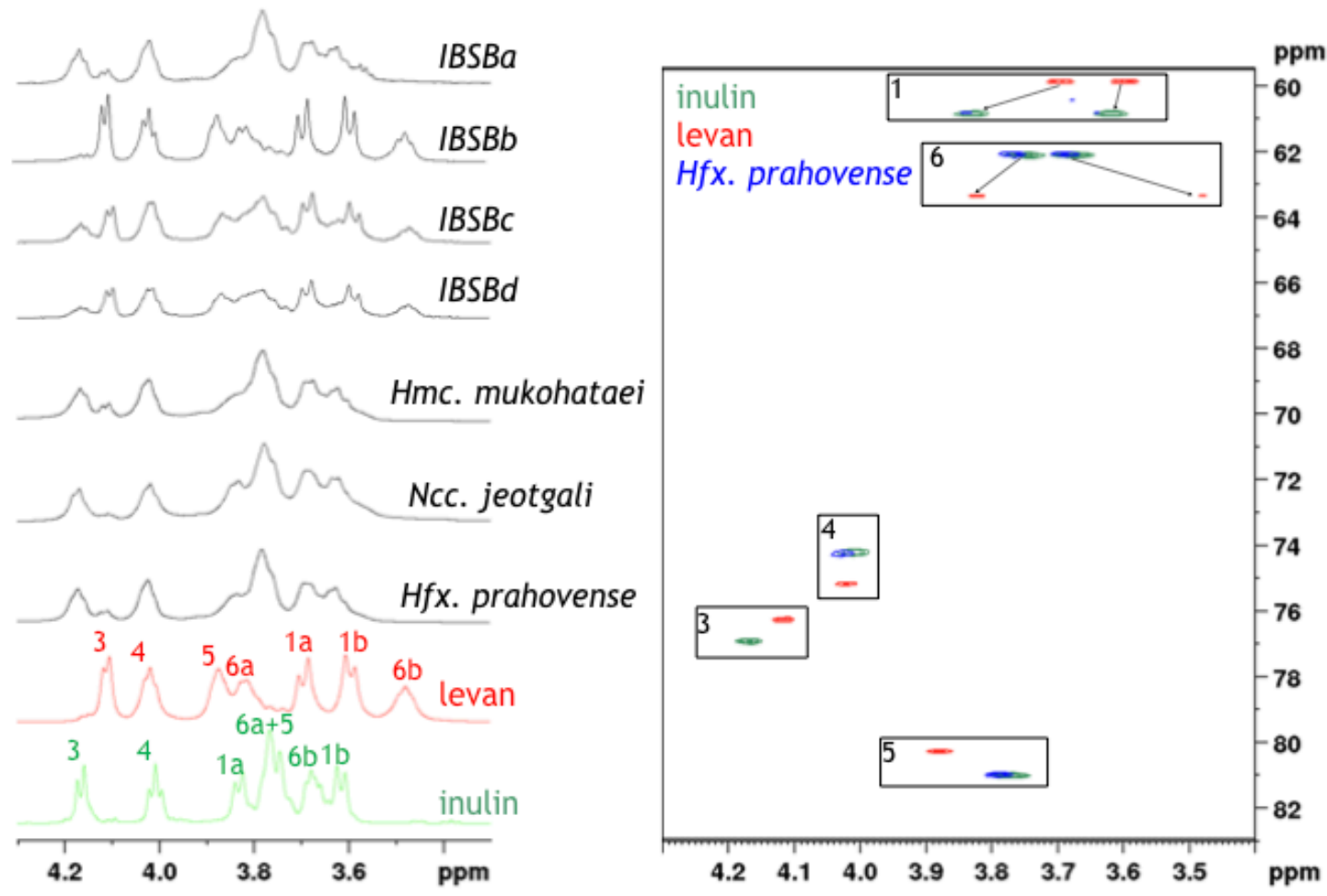

Fig. 2. NMR results of purified Archaeal fructans. Left: stacked plot of ${ }_{1}^{1}{ }^{1} \mathrm{H}$ spectra of reference samples and fructans isolated from a selection of Archaea, with strain and species names indicating their sources; right: overlay of main signals in the gHSQC of reference samples and the fructan isolated from $H f x$. prahovense DSM 18310 (blue), demonstrating match with inulin. Arrows indicate the typical downfield shift of ${ }^{13} \mathrm{C}$ atoms that are involved in a glycosidic linkage. Numbers refer to assignment of signals. Each box in the right panel includes signals with the same assignment.

Usually, the degree of branching in polysaccharides is estimated based on the relative intensity of well separated anomeric proton signals (Gidley, 1985; Nilsson, Gorton, Bergquist \& Nilsson, 1996; Zhu, 2017). However, fructans do not have a proton in this position. Therefore, we used the relative intensity of C6/H6 crosspeaks in the gHSQC that are well dispersed depending on the linkages in which fructose units are involved (Fig. 3). Branching percentage 
calculations based on the cross peak volumes of observed C6/H6 correlation in gHSQC revealed that fructans from Halobacteria are branched with varying degrees (Table 1). Among the fructans of Tuz Lake isolates, only levan from strain IBSBb could be used for branching calculations, because the other three showed too weak signals at relative positions, due to low sample concentrations.

Branching characteristics of levans and inulins depend greatly on their sources. For instance, bacterial inulins are more branched than plant inulins (Shoaib et al., 2016). Levans from Eubacteria exhibit varying branching degrees, too: while levans of Bacillus subtilis, $Z y$ momonas mobilis and Erwinia herbicola had branching degrees between 10-11\% (Benigar et al., 2014), levans obtained from the cultures of $H$. smyrnensis AAD6 (Poli et al., 2009) and levansucrase of Brenneria sp. EniD312 (Xu et al., 2018) were highly linear. These results also indicate that the conditions under which the polymerization of levan is carried out affect its branching characteristics. Halomonas levan used in this study was produced via the recombinant levansucrase of $H$. smyrnensis AAD6 (Kurtel et al., 2018b), and contrary to microbially-produced Halomonas levan, this enzymatically-produced levan had a branching degree of 10.08\% (Table 1). Except for Ncc. jeotgali inulin, fructans from Halobacteria used in this

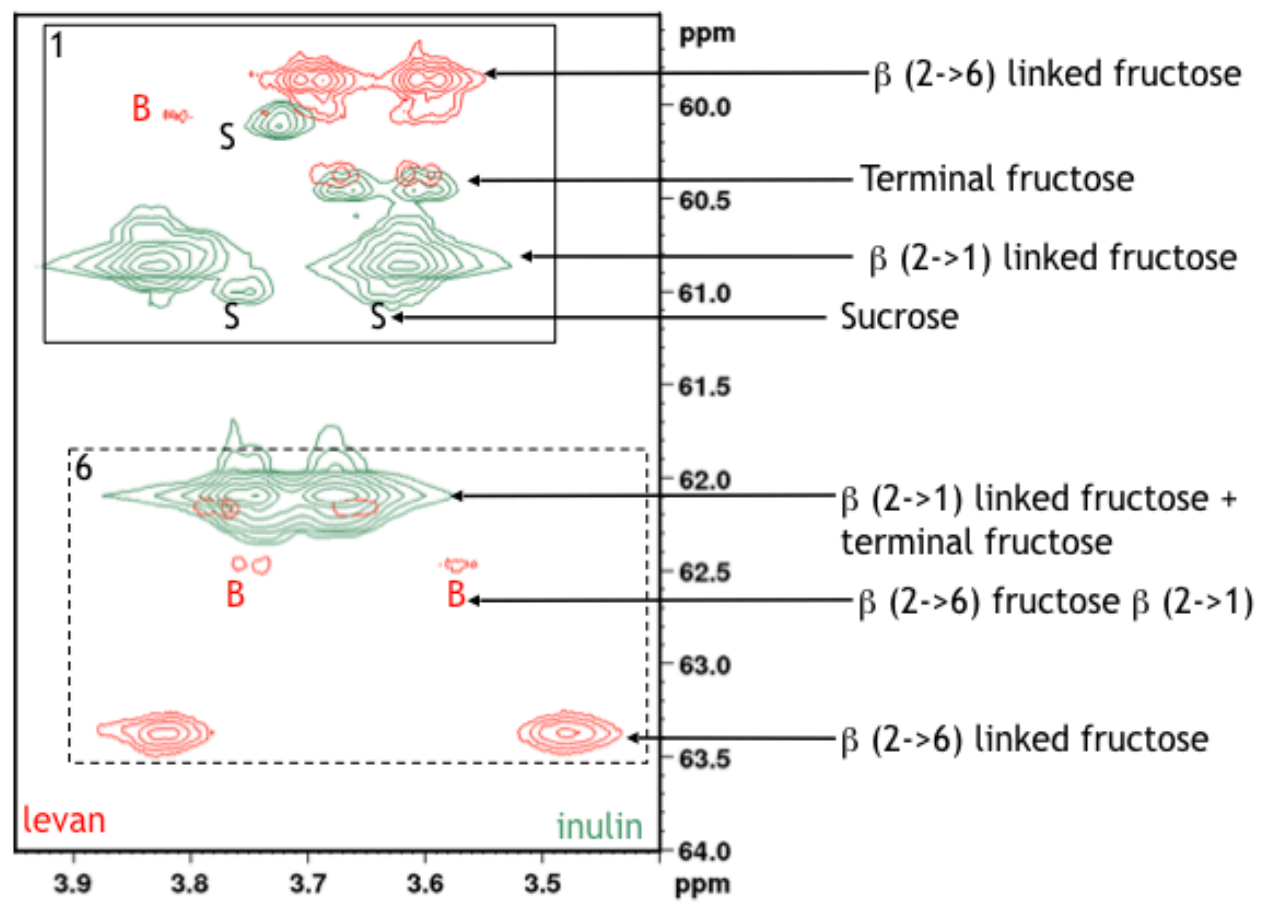


study had somewhat higher branching degrees compared to Eubacterial ones, indicating that Halobacterial fructans may have distinctive and novel physicochemical properties.

Fig. 3. Branching characteristics of levan and inulin. Section of gHSQC containing $\mathrm{C} 1$ and C6 signals at low threshold showing weaker signals of terminal fructose and fructose residues at a branchpoint (labeled with B). For inulin, also Suc signals are visible (labeled with S). Signals boxed in solid line: $1-\mathrm{CH}_{2}$; signals boxed in dashed line: $6-\mathrm{CH}_{2}$. Inulin reference sample was purchased from Orafti ${ }^{\circledR}$ (HP, plant origin, low DP), while levan was produced via recombinant levansucrase enzyme of H. smyrnensis AAD6 (high DP, Kırtel et al., 2018b).

Table 1. Branching in fructans used in this study based on the cross peak volumes (V) of observed C6/H6 correlation in gHSQC.

\begin{tabular}{|c|c|c|c|c|}
\hline Fructan & V (terminal) & $\mathbf{V}(\boldsymbol{\beta})$ & $V(2-6)$ & Branching (\%) \\
\hline Halomonas levan ${ }^{\mathrm{a}}$ & 26.04 & 23.45 & 183.11 & 10 \\
\hline \multirow[t]{2}{*}{ strain IBSBb levan } & 21.98 & 20.10 & 123.40 & 12 \\
\hline & $\mathbf{V}(\underset{(2-1)}{\text { terminal })}+$ & & & \\
\hline Hmc. mukohataei inulin & 329.36 & 61.99 & & 16 \\
\hline Hfx. prahovense inulin & 1003.90 & 170.90 & & 15 \\
\hline Ncc. jeotgali inulin & 180.60 & 19.10 & & 10 \\
\hline
\end{tabular}

aSynthesized via recombinant levansucrase enzyme of the bacterium $H$. smyrnensis AAD6 as reported in Kurtel et al. (2018b). 


\subsection{Taxonomic classification of Tuz Lake isolates}

The strains isolated from Tuz Lake were taxonomically classified according to their $16 \mathrm{~S}$ rRNA sequences as members of the genus Halomicrobium and they were named as Halomicrobium sp. IBSBa, IBSBb, IBSBc and IBSBd with respective GenBank accession numbers of MK208530, MK208531, MK208532 and MK208533. When these sequences were aligned using ClustalW, identity percentages between them were as follows (\%): IBSBa-IBSBb: 96, IBSBa-IBSBc: 97, IBSBa-IBSBd: 95, IBSBb-IBSBc: 99, IBSBb-IBSBd: 95, IBSBc-IBSBd: 96. NCBI BLAST analyses revealed that all Tuz Lake strains except for IBSBd showed a sequence identity of $>96 \%$ with Hmc. mukohataei DSM 12286, while for IBSBd the identity was $94 \%$. Taxonomically closest strains to our isolates were either Halomicrobium sp. A191 (DQ309091.1), which was also isolated from Tuz Lake, or Halomicrobium sp. HArcht1-2 (KT247947.1). A phylogenetic tree was constructed using the 16S rRNA gene sequences from various Archaea, both predicted to be fructanogenic or not (Fig. 4). As seen in the tree, several Halomicrobium strains appear closest to Tuz Lake isolates. Thus, the full lineage of the Tuz Lake isolates was determined as follows: Archaea; Euryarchaeota; Stenosarchaea group; Halobacteria; Halobacteriales; Haloarculaceae; Halomicrobium; Halomicrobium sp. IBSB.

According to NCBI Taxonomy database, currently there are only three identified species in the Halomicrobium genus; namely Halomicrobium mukohataei, Halomicrobium katesii and Halomicrobium zhouii. Members of this genus stain Gram-negative, and show red to orange-red pigmentation. They are all extreme halophiles, requiring at least $1.4-3.4 \mathrm{M}$ $\mathrm{NaCl}$ for growth, and have been isolated from various salt lakes, salterns and salt flats (Oren, 2015). Since Halomicrobium spp. typically carry multiple copies of divergent 16S rRNA genes (Cui et al. 2009), taxonomic identification on the species level requires extensive work.

As discussed in Kırtel et al. (2018a), putative GH-J clan genes are present in only extremely halophilic class of Halobacteria within all Archaeal lineages. Fructanogenic Archaea show a wide distribution within all three orders of the Halobacteria class (Fig. 4), dismissing 
the possibility that fructan synthesis is a rare, exceptional phenomenon in Halobacteria. As revealed in the phylogenetic tree, both fructanogenic and non-fructanogenic species are commonly encountered within the same genera. In the Halobacteriales order, 17 different species were predicted to harbor putative GH68 family genes, with the majority belonging to the Haloarcula genus; a close relative of the genus Halomicrobium. In the order of Haloferacales, fructanogenic species show a wider distribution with 32 different species, 12 of them belonging to the Halorubrum genus. As for the Natrialbales order, only 9 species in the genera of Halostagnicola, Haloterrigena, Natrialba and Natronococcus carry putative GH68 family genes. Thus, approximately $21 \%, 31 \%$ and $16 \%$ of all known species of Halobacteriales, Haloferacales and Natrialbales orders, respectively, were predicted to be fructanogenic. 


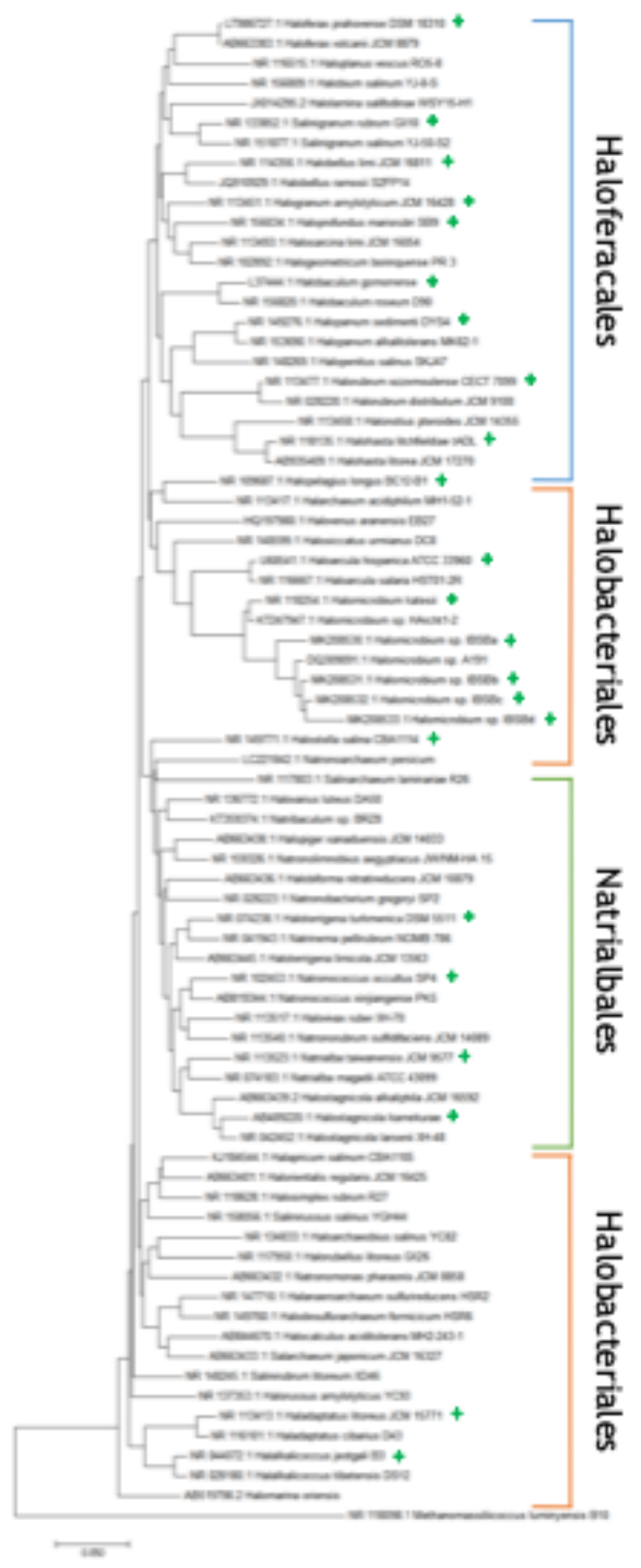

Fig. 4. Taxonomic distribution of fructanogenic Archaea. Distribution of some Archaea that harbor putative GH68 family genes within the Halobacteria class, each denoted with a star. The phylogenetic tree was based on 16S rRNA gene sequences of some Archaea from all three orders of the Halobacteria class, and built with MEGA7 (Kumar, Stecher, \& Tamura, 2016) using neighbor-joining method. Methanomassilicoccus luminyensis B10 was used as the outgroup.

As discussed by Méheust et al. (2018), hundreds of chimeric protein families including GH-J clan enzymes in Halobacteria are most probably of Eubacterial origin, occuring as a result of gene transfer events throughout their evolution. This enabled Halobacteria to adapt 
to an aerobic lifestyle, unlike other classes of the archaeal domain of life, thus giving them the ability to utilize a wide variety of carbohydrates via aerobic respiration. Absence of any GH-J clan gene in methanogenic Archaea, which are the closest relatives and ancestors of Halobacteria, support the possibility that fructan metabolism appeared in Halobacteria in parallel with the emergence of their aerobic capabilities.

\subsection{Structural investigation into Halobacterial GH-J clan enzymes}

All the type strains used in this study, Hmc. mukohataei, Hfx. prahovense and Ncc. jeotgali, harbor one GH68 gene and one GH32 gene each. Multiple alignment and homology modelling analyses of these GH68 protein sequences via NCBI-COBALT (Papadopoulos \& Agarwala, 2007) and SWISS-MODEL (Biasini et al., 2014) were carried out. Multiple alignment of these four Halobacterial GH68 enzymes with some of their well-characterized Eubacterial homologues revealed that all critical amino acid residues that take part in catalysis are strictly conserved (Fig. 5). Numbered according to their homologues in H. smyrnensis AAD6 levansucrase, these residues are as follows: D47 (the nucleophile), W130 (plays a role in substrate binding), R201 (suggested to form a salt bridge with E286, Martínez-Fleites et al., 2005), D2O2 (the transition-state stabilizer), E286 (general acid/base catalyst) and H304 (crucial for the polymerization process). These residues were also evaluated for their positions and they were all observed to be superimposed towards the substrate located inside the deep central cavity of the 5-bladed $\beta$-propeller domain. Interestingly, although they all produce inulin, these Halobacterial GH68 enzymes show higher sequence similarity with known levansucrases (around 35\%) compared to inulosucrases (around 25\% for inulosucrase from Lactobacillus johnsonii, PDB ID: 2YFR), even with homologues of all the above-mentioned amino acid residues being also strictly conserved in inulosucrases. In fact, the mechanism that determines whether a fructan produced by the enzyme will carry either $\beta$-2,6 (levantype) or $\beta-2,1$ (inulin-type) fructofuranosidic linkages on its backbone is still not understood and these Halobacterial enzymes may be the key to resolve this issue in the future. 


\begin{tabular}{|c|c|c|c|c|c|c|c|c|c|}
\hline & 1 & MHD [ & 5]RPG & & & RR-GERA $\quad$ - & -VAPVVYPPAEDVDP & & 58 \\
\hline & 1 & & & -- IPA--. & -WTREHAESI & RR--DDS & -VAPVIYPPDERLDD & DLHIWDTWFLRNR & 53 \\
\hline H.mukohataei & 1 & MSK [ & 2]-PG & TAVPGhgArS & GWSREQASRI & ER--TDDT & -TAPIVYPPATDQAP & DVHVWDTWLLRER & 60 \\
\hline H. smyrnensis & 1 & --- & MSI & QDKIQ--T-A & SWSRADALKV & TF--DDPT & TTQPEVGYDFPVLDD & DLFIWD'TMPLRNL & 54 \\
\hline E. amylovora & 1 & --- & MSD & YNYKP--T-L & -WTRADALKV & $\mathrm{HE}--\mathrm{DDPT}$ & TTQPVIDIAFPVMSE & EVFIWDTMPLRDF & 53 \\
\hline G. diazotrophicus & & --- & $---[16]$ & ] YDPQS S f T $-\mathrm{A}$ & RWTRADALQI & KAhs DATV [10] & ] LTMPNI PADFPVINP & DVWVWDTWTLIDK & 81 \\
\hline B.subtilis & & & & KETYG--I-S & HITRHDMLQI & $\mathrm{PE}-\mathrm{Q}$ QRKN [1 & ] STIKNISSAKG---- & -LDVWD'SWPLQNA & \\
\hline sonii & & 27] AEN & & ] FSKEAkSG- & QLTYNDEKKI & & & ] DLEIWD & \\
\hline gali & 59 & -GSVAEI & GYRVILSI & LTAPAELLP & GKRHDVAT & IRYFYSRDGR & EWTCGGRVEEEGAAFG & SROWAGSALYD & 127 \\
\hline & 54 & -GSVAEI & DGYRVVFSI & LTSSSDLLP & GKRHDVAT & IRYFYSRDGE & EWTTGGVVFD-GGALG & $Q R Q \overline{\mathbf{W}} A$ & 121 \\
\hline H.mukohataei & 61 & -GTVATV & DGYRVTFSI & LTAPADLIP & GKRHDVAT & IRYFYSADGR & TWOPGGVVFE-E-PLG & ORTW $\bar{W} A G S A L Y D$ & 127 \\
\hline & 55 & -GDVYSY & DGWSITFTI & "LTADRKPE- $[1$ & 51 TDRHGRAR & MCYWYAKDSK & SWTFGGRYMOEGVS-- & 1 1TRE & 137 \\
\hline & 5 & IIs & $c$ & & 6] EDRH & & & & 38 \\
\hline phicus & 82 & ADQFSY & & & 2] DDRHVI & IGFFYRRAGI [ 9 & & RLM & 170 \\
\hline B.st & 61 & GTVANY & AGYHIVFAI & LAGDPK--- & --NADDTS & IYMFYQKVGE [ & SDKKFD [ & [9] TQE $\overline{\mathbf{W}} z$ & 137 \\
\hline . joh & 137 & tGYVSNW & NGYQLVIGN & GMMGVPNVND & $-----\mathrm{NH}$ & IYLLYNKYGD [ 4 & 4] HWKNAGPIFGLGTPVI & -QQW̄SGSATLN & 203 \\
\hline & & & & & & & & & \\
\hline & 12 & & & & & & & & 193 \\
\hline & & & & & & & & RGM & 194 \\
\hline H.st & 138 & DKGD & YYTAV- & -TPGA-- & $-\cdots-$ TMA $_{1}$ & G--RIVTTE & ETEVE & DGVYYQT & 197 \\
\hline & 13 & & & & & & & & 198 \\
\hline ophicus & 171 & $\mathrm{Q}[1] \mathrm{HC}$ & & & & & & $Q N$ & 243 \\
\hline & 138 & & & & 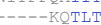 & & & & 201 \\
\hline & 204 & 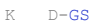 & & & & & QDKIS & & 271 \\
\hline & & & & & & & & & \\
\hline & 19 & & & & & & & --FNG! & 245 \\
\hline & $1 \mathrm{~s}$ & & & & & & & $----F N G$ & 247 \\
\hline & 19 & $-\mathrm{TNE}$ & & & GNIG & & & GARFQSGC & 261 \\
\hline & 19 & & & & & SHE & & & 262 \\
\hline & & & & & & & & & \\
\hline & 2 & ] $\mathrm{NH}$ & & & & & & & 2 \\
\hline & 272 & & & & & WLN & NLGDFFQ & I I KKL & 354 \\
\hline & & & & & & & & & \\
\hline & & & & & & YHLF & & & 318 \\
\hline & & & & & & & & & \\
\hline & & & & & & & & & \\
\hline & & & & & & & & & 35 \\
\hline
\end{tabular}

\begin{tabular}{|c|c|c|c|c|c|c|c|c|}
\hline G.diazotrophicus & 316 & \multicolumn{4}{|c|}{ TDSTL--SKWKFLSPLISANCVNDQTERPQVYLHNGKYYIFTISHRTTFA } & \multicolumn{2}{|c|}{ AGVDGPDGVY-GFVGDGIRSDFQPMN $Y$} & 389 \\
\hline B.subtilis & 286 & \multicolumn{6}{|c|}{ 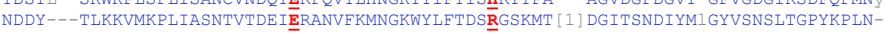 } & 359 \\
\hline L. johnsonii & 355 & \multicolumn{6}{|c|}{ NDDVKnPSVAKVYSPLISAPMVSDEI I- RPDVVKLGNKYYLFAAT站NRGS [ 8 ] NKAVGDNVAMiGYVSDNLTHGYVPLN- } & 438 \\
\hline N. jeotgali & 334 & \multirow{2}{*}{$\begin{array}{l}\text { ESGLVLTNP--ES } \\
\text { GHGMVLTNP--KG }\end{array}$} & & \multicolumn{2}{|c|}{ PFQTYSWLAYPHDEEVLVTSFFNYYDLRGLS } & \multirow{2}{*}{\multicolumn{2}{|c|}{ LD [11] RRFG--GTLAPTVRLGVDG }} & 406 \\
\hline H.prahovense & 319 & & A & \multirow{2}{*}{\multicolumn{2}{|c|}{ PFQAYSWLVYDHGDDLLVSSFFNYFDYDRPS }} & LD [ : & & 391 \\
\hline H.mukohataei & 321 & DSGLVVTNP--AN & A & & EADS & 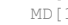 & ] RRFG--GTLAPTLRLRVEG & 393 \\
\hline H. smyrnensis & 335 & GSGLVLGNP--SS & $Q$ & \multicolumn{2}{|c|}{ PFOTYSHCVMPN---GLVTSFIDSVEKGGKT } & ED & YRIG--GTEAPTLELKIEG & 393 \\
\hline E. amylovora & 336 & SSGLVLGNP--SS & S $Q$ & \multirow{2}{*}{\multicolumn{2}{|c|}{ 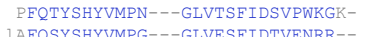 }} & $-D$ & YRIG--GTEAPTVKILLKG & 392 \\
\hline G.diazotrophicus & 390 & \multirow{2}{*}{$\begin{array}{l}\text { GSGLTMGNPtdLN } \\
\text { KTGLVLKMDIdPN }\end{array}$} & $\mathrm{N}[4] \mathrm{T}[11$ & & & -- & $---G--G T L A P T V R V R I A Q$ & 458 \\
\hline B. subtilis & 360 & & N D & VTETYSHFAVPQ-AKGNNVVITSYMTI & JRGEY & -- & ADKQ--STFAPSFLINIKG & 420 \\
\hline L. johnsonii & 439 & \multicolumn{2}{|c|}{ ESGVVLTASVPAN [2] - } & \multicolumn{2}{|c|}{-TATYSYYAVPVEGRDDQLLITSYITNRGEV } & -- & AGKGmhATWAPSFLLQINP & 502 \\
\hline$N \cdot j e$ & 407 & TETRVI & HGHLP & & 444 & & & \\
\hline H.prahove & 392 & DETDVLGTLG & HGHLP & LPRETLPTPWWER-DGDATRGG [2] & 429 & & & \\
\hline H.muko & 394 & THTEILGTLD & HWQIP & LPDEVLPPTDREY fAGESGDGG [ 6$]$ & 436 & & & \\
\hline H.smyrnensis & 394 & NNTYVVKEYD & YGEIP & k-..-.-.-- & 416 & & & \\
\hline E. amylovora & 393 & DRSFIVDSED & YGYIP & AMKDITLK------- & 415 & & & \\
\hline G.diazotrophicus & 459 & NASAVDLRYG [ 6 ] & ] YGDIP [1 & 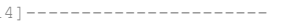 & 493 & & & \\
\hline B. subti & 421 & & QGQLT & & & & & \\
\hline L. johnsonii & 503 & NTTTVLAKM | & 10 GDWI & ] LPGEWGKPVDWDLI GGYNLKPH [ 7] & 571 & & & \\
\hline
\end{tabular}

Fig. 5. Constraint-based multiple sequence alignment of several GH68 family enzymes.

Alignment of Halobacterial GH68 family enzymes (with accession numbers: ELY66219.1 from Ncc. 
jeotgali, ELZ65257.1 from Hfx. prahovense, and ACV48051.1 from Hmc. mukohataei) with their selected Eubacterial homologues (levansucrase from H. smyrnensis AAD6, accession number: AGG11046.1; levansucrase from E. amylovora, PDB ID: 4D47; levansucrase from Gluconacetobacter diazotrophicus, PDB ID: 1W18; levansucrase from Bacillus subtilis, PDB ID: 2VDT; inulosucrase from Lactobacillus johnsonii, PDB ID: 2YFR). The alignment was carried out via NCBI COBALT. Conservation setting was set to 4 . Bold red letters indicate strictly conserved amino acids that take part in catalysis.

\section{Conclusions}

The exact physiological functions of fructans in Halobacteria are yet unkown. Fructan and fructan metabolizing localization studies are required to shed light on this. The fact that our isolated strains can also grow on minimal media without Suc suggests that fructan formation is not strictly required for survival. Haloarcheae are strictly heterotrophic and therefore Suc, the substrate for fructan synthesis, should have an exogenous origin. To shed light upon this subject, future studies should specifically focus on halophilic Suc-producers in hypersaline environments (Oren, 2015). For our four fructanogenic isolates, from Tuz lake, a rather "closed" ecosystem almost entirely fed by rain and ground water (Kashima, 2002), we expect that only one or a few autotrophic (Suc-producing) microorganism(s) are required to deliver Suc (Oren, 2014).

The fructanogenic character appeared in Halobacterial members isolated from different places all over the world and the encoding GH-J genes seem to be widespread among Halobacteria, suggesting that fructans may provide benefits in hypersaline environments where Suc is available. We also detected a different branching degree on native Halobacterial levan, and these distinctive and novel physicochemical properties are promising in terms of biotechnological applications. Future studies will involve obtaining the whole genome sequences of Tuz Lake isolates to get more solid information on Archaeal FTs. Overall, this work sets a milestone for carbohydrate/protein science in Archaea. Also, deeper insights into novel halophilic enzymes may provide strategies that help to overcome salinization, a serious threat to 
all life forms in the future. Now that it is demonstrated here that fructans are present in all three domains of life, in the future these polymers may prove to be key molecules to reveal yet-unknown dynamics shaping life in the presence of (salt and) Suc.

\section{Acknowledgements}

\section{Declarations of interest: None.}

Funding sources: This research did not receive any specific grant from funding agencies in the public, commercial, or not-for-profit sectors.

\section{References}

Adamberg, K., Tomson, K., Talve, T., Pudova, K., Puurand, M., Visnapuu, T., ... \& Adamberg, S. (2015). Levan enhances associated growth of Bacteroides, Escherichia, Streptococcus and Faecalibacterium in fecal microbiota. PloS One, 10, e0144042.

Avila-de Dios, E., Gomez-Vargas, A. D., Damian-Santos, M. L., \& Simpson, J. (2015). New insights into plant glycoside hydrolase family 32 in Agave species. Frontiers in Plant Science, 6, 594 .

Benigar, E., Dogsa, I., Stopar, D., Jamnik, A., Cigić, I. K., \& Tomšič, M. (2014). Structure and dynamics of a polysaccharide matrix: aqueous solutions of bacterial levan. Langmuir, $30,4172-4182$.

Biasini, M., Bienert, S., Waterhouse, A., Arnold, K., Studer, G., Schmidt, T., ... \& Schwede, T. (2014). SWISS-MODEL: modelling protein tertiary and quaternary structure using evolutionary information. Nucleic Acids Research, 42, W252-W258.

Borrel, G., McCann, A., Deane, J., Neto, M. C., Lynch, D. B., Brugère, J. F., \& O'Toole, P. W. (2017). Genomics and metagenomics of trimethylamine-utilizing Archaea in the human gut microbiome. The ISME Journal, 11, 2059-2074. 
Choukade, R., \& Kango, N. (2019). Characterization of a mycelial fructosyltransferase from Aspergillus tamarii NKRC 1229 for efficient synthesis of fructooligosaccharides. Food Chemistry, 286, 434-440.

Cui, H. L., Zhou, P. J., Oren, A., \& Liu, S. J. (2009). Intraspecific polymorphism of 16S rRNA genes in two halophilic archaeal genera, Haloarcula and Halomicrobium. Extremophiles, 13, 31-37.

Dogsa, I., Brloznik, M., Stopar, D., \& Mandic-Mulec, I. (2013). Exopolymer diversity and the role of levan in Bacillus subtilis biofilms. PloS One, 8, e62044.

Enache, M., Itoh, T., Kamekura, M., Teodosiu, G., \& Dumitru, L. (2007). Haloferax prahovense sp. nov., an extremely halophilic archaeon isolated from a Romanian salt lake. International Journal of Systematic and Evolutionary Microbiology, 57, 393-397.

Gaci, N., Borrel, G., Tottey, W., O’Toole, P. W., \& Brugère, J. F. (2014). Archaea and the human gut: new beginning of an old story. World Journal of Gastroenterology: WJG, 20, 16062.

Gidley, M. J. (1985). Quantification of the structural features of starch polysaccharides by NMR spectroscopy. Carbohydrate Research, 139, 85-93.

Hardoim, P. R., Van Overbeek, L. S., Berg, G., Pirttilä, A. M., Compant, S., Campisano, A., ... \& Sessitsch, A. (2015). The hidden world within plants: ecological and evolutionary considerations for defining functioning of microbial endophytes. Microbiology and Molecular Biology Reviews, 79, 293-320.

Ihara, K., Watanabe, S., \& Tamura, T. (1997). Haloarcula argentinensis sp. nov. and Haloarcula mukohataei sp. nov., two new extremely halophilic archaea collected in Argentina. International Journal of Systematic and Evolutionary Microbiology, 47, 73-77.

Kang, S. A., Jang, K. H., Seo, J. W., Kim, K. H., Kim, Y. H., Rairakhwada, D., ... \& Rhee, S. K. (2009). Levan: applications and perspectives. In Microbial Production of Biopolymers and Polymer Precursors: Applications and Perspectives, 145-161. 
Kashima, K. (2002). Environmental and climatic changes during the last 20,000 years at Lake Tuz, central Turkey. Catena, 48, 3-20.

Kırtel, O., Versluys, M., Van den Ende, W., \& Toksoy Öner, E. (2018a). Fructans of the saline world. Biotechnology Advances, 36, 1524-1539.

Kırtel, O., Menéndez, C., Versluys, M., Van den Ende, W., Hernández, L., \& Toksoy Öner, E. (2018b). Levansucrase from Halomonas smyrnensis AAD6T: first halophilic GH-J clan enzyme recombinantly expressed, purified, and characterized. Applied Microbiology and Biotechnology, 102, 9207-9220.

Koczan, J. M., McGrath, M. J., Zhao, Y., \& Sundin, G. W. (2009). Contribution of Erwinia amylovora exopolysaccharides amylovoran and levan to biofilm formation: implications in pathogenicity. Phytopathology, 99, 1237-1244.

Kumar, S., Stecher, G., \& Tamura, K. (2016). MEGA7: molecular evolutionary genetics analysis version 7.0 for bigger datasets. Molecular Biology and Evolution, 33, 1870-1874.

Lammens, W., Le Roy, K., Schroeven, L., Van Laere, A., Rabijns, A., \& Van den Ende, W. (2009). Structural insights into glycoside hydrolase family 32 and 68 enzymes: functional implications. Journal of Experimental Botany, 6o, 727-740.

Lei, X. J., Kim, Y. M., Park, J. H., Baek, D. H., Nyachoti, C. M., \& Kim, I. H. (2017). Effects of levan-type fructan on growth performance, nutrient digestibility, diarrhoea scores, faecal shedding of total lactic acid bacteria and coliform bacteria, and faecal gas emission in weaning pigs. Journal of The Science of Food and Agriculture, 98, 1539-1544.

Loukas, A., Kappas, I., \& Abatzopoulos, T. J. (2018). HaloDom: a new database of halophiles across all life domains. Journal of Biological Research-Thessaloniki, 25, 2.

Martínez-Fleites, C., Ortíz-Lombardía, M., Pons, T., Tarbouriech, N., Taylor, E. J., Arrieta, J. G., ... \& Davies, G. J. (2005). Crystal structure of levansucrase from the Gram-negative bacterium Gluconacetobacter diazotrophicus. Biochemical Journal, 39o, 19-27. 
Méheust, R., Watson, A. K., Lapointe, F. J., Papke, R. T., Lopez, P., \& Bapteste, E. (2018). Hundreds of novel composite genes and chimeric genes with bacterial origins contributed to haloarchaeal evolution. Genome Biology, 19, 75.

Nilsson, G. S., Gorton, L., Bergquist, K. E., \& Nilsson, U. (1996). Determination of the Degree of Branching in Normal and Amylopectin Type Potato Starch with 1H-NMR Spectroscopy: Improved resolution and two-dimensional spectroscopy. Starch-Stärke, 48, 352-357.

Orellana, L. H., Chee-Sanford, J. C., Sanford, R. A., Löffler, F. E., \& Konstantinidis, K. T. (2018). Year-round shotgun metagenomes reveal stable microbial communities in agricultural soils and novel ammonia oxidizers responding to fertilization. Applied and Environmental Microbiology, 84, e01646-17.

Oren, A., Elevi, R., Watanabe, S., Ihara, K., \& Corcelli, A. (2002). Halomicrobium mukohataei gen. nov., comb. nov., and emended description of Halomicrobium mukohataei. International Journal of Systematic and Evolutionary Microbiology, 52, 1831-1835.

Oren, A. (2008). Microbial life at high salt concentrations: phylogenetic and metabolic diversity. Saline Systems, 4, 2.

Oren, A. (2014). The ecology of Dunaliella in high-salt environments. Journal of Biological Research-Thessaloniki, 21, 23.

Oren, A. (2015). Cyanobacteria in hypersaline environments: biodiversity and physiological properties. Biodiversity and Conservation, 24, 781-798.

Oren, A. (2016). Halomicrobium. Bergey's Manual of Systematics of Archaea and Bacteria, 1-8. John Wiley \& Sons, Inc.

Papadopoulos, J. S., \& Agarwala, R. (2007). COBALT: constraint-based alignment tool for multiple protein sequences. Bioinformatics, 23, 1073-1079.

Peshev, D., \& Van den Ende, W. (2014). Fructans: prebiotics and immunomodulators. Journal of Functional Foods, 8, 348-357. 
Poli, A., Kazak, H., Gürleyendă̆, B., Tommonaro, G., Pieretti, G., Toksoy Öner, E., \& Nicolaus, B. (2009). High level synthesis of levan by a novel Halomonas species growing on defined media. Carbohydrate Polymers, 78, 651-657.

Roh, S. W., Nam, Y. D., Chang, H. W., Sung, Y., Kim, K. H., Lee, H. J., ... \& Bae, J. W. (2007). Natronococcus jeotgali sp. nov., a halophilic archaeon isolated from shrimp jeotgal, a traditional fermented seafood from Korea. International Journal of Systematic and Evolutionary Microbiology, 57, 2129-2131.

Schleucher, J., Schwendinger, M., Sattler, M., Schmidt, P., Schedletzky, O., Glaser, S. J., ... \& Griesinger, C. (1994). A general enhancement scheme in heteronuclear multidimensional NMR employing pulsed field gradients. Journal of Biomolecular NMR, 4, 301-306.

Sehgal, S. N., \& Gibbons, N. E. (1960). Effect of some metal ions on the growth of $\mathrm{Ha}^{-}$ lobacterium cutirubrum. Canadian Journal of Microbiology, 6, 165-169.

Shoaib, M., Shehzad, A., Omar, M., Rakha, A., Raza, H., Sharif, H. R., ... \& Niazi, S. (2016). Inulin: Properties, health benefits and food applications. Carbohydrate Polymers, $147,444-454$.

Tarkowski, Ł. P., Van de Poel, B., Höfte, M., \& Van den Ende, W. (2019). Sweet Immunity: Inulin Boosts Resistance of Lettuce (Lactuca sativa) against Grey Mold (Botrytis cinerea) in an Ethylene-Dependent Manner. International Journal of Molecular Sciences, 20, 1052.

Toksoy Öner, E. T., Hernández, L., \& Combie, J. (2016). Review of levan polysaccharide: from a century of past experiences to future prospects. Biotechnology Advances, 34, 827844 .

Vanhaecke, M., Van den Ende, W., Van Laere, A., Herdewijn, P., \& Lescrinier, E. (2006). Complete NMR characterization of lychnose from Stellaria media (L.) Vill. Carbohydrate Research, 341, 2744-2750. 
Verspreet, J., Hansen, A. H., Dornez, E., Delcour, J. A., Van den Ende, W., Harrison, S. J., \& Courtin, C. M. (2015). LC-MS analysis reveals the presence of graminan-and neo-type fructans in wheat grains. Journal of Cereal Science, 61, 133-138.

Wei, H., Zhao, H., Su, T., Bausewein, A., Greiner, S., Harms, K., \& Rausch, T. (2017). Chicory R2R3-MYB transcription factors CiMYB5 and CiMYB3 regulate fructan 1-exohydrolase expression in response to abiotic stress and hormonal cues. Journal of Experimental Botany, 68, 4323-4338.

Woese, C. R., Kandler, O., \& Wheelis, M. L. (1990). Towards a natural system of organisms: proposal for the domains Archaea, Bacteria, and Eucarya. Proceedings of the National Academy of Sciences, 87, 4576-4579.

Xu, X., Gao, C., Liu, Z., Wu, J., Han, J., Yan, M., \& Wu, Z. (2016). Characterization of the levan produced by Paenibacillus bovis sp. nov BD3526 and its immunological activity. Carbohydrate Polymers, 144, 178-186.

Xu, W., Liu, Q., Bai, Y., Yu, S., Zhang, T., Jiang, B., \& Mu, W. (2018). Physicochemical properties of a high molecular weight levan from Brenneria sp. EniD312. International Journal of Biological Macromolecules, 109, 810-818.

Zannini, E., Waters, D. M., Coffey, A., \& Arendt, E. K. (2016). Production, properties, and industrial food application of lactic acid bacteria-derived exopolysaccharides. Applied Microbiology and Biotechnology, 10o, 1121-1135.

Zaremba-Niedzwiedzka, K., Caceres, E. F., Saw, J. H., Bäckström, D., Juzokaite, L., Vancaester, E., ... \& Stott, M. B. (2017). Asgard archaea illuminate the origin of eukaryotic cellular complexity. Nature, 541, 353-358.

Zhang, J., Liu, C., Xie, Y., Li, N., Ning, Z., Du, N., ... \& Zhong, Y. (2017). Enhancing fructooligosaccharides production by genetic improvement of the industrial fungus Aspergillus niger ATCC 20611. Journal of Biotechnology, 249, 25-33.

Zhu, F. (2017). NMR spectroscopy of starch systems. Food Hydrocolloids, 63, 611-624. 